\title{
Clinical course of thoracic mycotic aneurysm in a patient who was rescued by thoracic endovascular aortic repair with video-assisted thoracic surgery after rupture
}

\author{
Yun Seok $\mathrm{Kim}^{1}$ \\ ${ }^{1}$ Keimyung University Dongsan Hospital
}

March 28, 2021

\begin{abstract}
Ruptured thoracic mycotic aneurysm is fatal, and surgical resection of the aorta may be unavailable because of occurrence of preoperative shock in such a patient. In the present case, the thoracic mycotic aneurysm ruptured during hospital stay, causing acute hypovolemic shock but was successfully repaired by thoracic endovascular aortic repair (TEVAR) with video-assisted thoracic surgery (VATS) drainage.
\end{abstract}

Clinical course of thoracic mycotic aneurysm in a patient who was rescued by thoracic endovascular aortic repair with video-assisted thoracic surgery after rupture

Yun Seok Kim ${ }^{1}$

${ }^{1}$ Department of Thoracic and Cardiovascular surgery, Keimyung University Dongsan Hospital, Daegu, Republic of Korea

Corresponding author

Yun Seok Kim, MD, PhD

Department of Thoracic and Cardiovascular Surgery, Keimyung University Dongsan Hospital, 1035, Dalgubeol-daero, Dalseo-gu, Daegu, Republic of Korea.

E-mail:yunseok99@hanmail.net, Tel: 82-53-258-4783.

Word count: 994 words

\section{Abstract}

Ruptured thoracic mycotic aneurysm is fatal, and surgical resection of the aorta may be unavailable because of occurrence of preoperative shock in such a patient. In the present case, the thoracic mycotic aneurysm ruptured during hospital stay, causing acute hypovolemic shock but was successfully repaired by thoracic endovascular aortic repair (TEVAR) with video-assisted thoracic surgery (VATS) drainage.

Key words: Mycotic aortic aneurysm, Thoracic endovascular aortic repair, Video-assisted thoracic surgery.

\section{Case}

A 76-year-old man presented to our emergency department complaining of febrile sensation since 9 days. The initial blood pressure was $180 / 100 \mathrm{mmHg}$ and heart rate was 115 beats per minute. His body temperature was $38.6^{\circ} \mathrm{C}$, white blood cell count was 19,410 cells/ $\mu \mathrm{L}$, and C-reactive protein (CRP) was 31.0 $\mathrm{mg} / \mathrm{dL}$. Computed tomography $(\mathrm{CT})$ revealed a pseudoaneurysm at the descending thoracic aorta, which 
was consistent with mycotic aneurysm (Figure 1-A). Empirical antibiotic therapy was initiated (vancomycin $1 \mathrm{~g}$ IV q $12 \mathrm{~h}$, piperacillin/tazobactam 4.5g IV q 8 hours) immediately after drawing two pairs of peripheral blood samples for culture.

Surgical aortic replacement or thoracic endovascular aortic repair (TEVAR) were recommended, but the patient delegated the decision to his family, who refused these procedures. Despite the empirical antibiotic therapy, intermittent fever continued. After 4 days, the patient complained of dyspnea, and chest X-ray revealed a large pleural effusion (Figure 2-HD5). Blood pressure was 129/61 $\mathrm{mmHg}$ and heart rate was 96 beats per minute at that time. CT revealed no evidence of contrast leakage into the pleural space (Figure 1-B). Therefore, a closed thoracostomy was performed, and $1200 \mathrm{~mL}$ of yellowish exudative effusion was drained. Following this, the patient remained stable. On the same day, blood culture revealed Streptococcus pneumoniae, and antibiotic therapy was adjusted (vancomycin 1g IV q 12 hours, ceftriaxone 2g IV q 14 hours).

Next day, more than $2 \mathrm{~L}$ of fresh blood was drained abruptly and the patient developed acute hypovolemic shock. Following the family's informed consent the patient proceeded for emergency TEVAR. This was performed successfully using a Seal Thoracic Stent Graft $34 \times 120 \mathrm{~mm}$ (S\&G Biotech, Yongin-si, South Korea); however, a large hematoma and ruptured abscess remained in the left pleural space, causing mediastinal shifting (Figure 2-HD6, post-TEVAR). Therefore, video-assisted thoracic surgery (VATS) was performed to remove hematoma and ruptured abscess and two plastic tubes remained for drainage. Four days later, the patient was transferred to the general ward. Further blood culture revealed no microorganisms. Vancomycin was discontinued on day 41 of hospitalization. On day 62, intravenous ceftriaxone was changed to oral cefpodoxime $(100 \mathrm{mg}$ bid), and the patient was discharged without complications. Cefpodoxime was maintained for 6 months. At the latest follow-up, 1 year after TEVAR, he remained stable without any signs of infection, and $\mathrm{CT}$ revealed no stent-related complications.

\section{Discussion}

Thoracic mycotic aneurysm has a high risk of rupture, and the mortality rate during medical treatment is about $60 \%$ (1). Open surgical repair can eliminate infected tissue and enable effective drainage. Despite this advantage, however, open surgical repair has a mortality rate of $12 \%$ to $36 \%(1,2)$, and would be much higher in cases of preoperative rupture causing hypovolemic shock. TEVAR has therefore been used to overcome high surgical mortality. Most reported cases, however, were 'impending rupture' or 'contained rupture' (3-5) but not true rupture causing massive hemothorax and hypovolemic shock. In the present case, the patient and his family refused invasive procedures, resulting in aortic rupture causing abrupt hypovolemic shock. Therefore, the clinical course of thoracic mycotic aneurysm could be observed.

Concerns about TEVAR in mycotic aneurysms remain because it does not enable complete resection of the infected tissue. Hirano et al. reported the use of a combination of TEVAR and VATS for debridement with good result (3). They performed VATS to resect and drain the infected aneurysm 2 weeks after TEVAR. In the present case, however, VATS was performed immediately after TEVAR because the mycotic aneurysm had already ruptured. Therefore we didn't need to perform further abscess drainage procedure.

In conclusion, TEVAR combined with VATS may be a viable option, especially in cases of ruptured mycotic aneurysm causing massive hemothorax and hypovolemic shock.

No funding source.

No conflicts of interest.

\section{Figure legends}

Figure 1. Initial CT scan reveals *contained rupture of thoracic mycotic aneurysm (A). After 4 days, there was a large newly developed ${ }^{+}$exudative pleural effusion but no evidence of contrast leakage into the pleural space (B). 
Figure 2. Consecutive chest X-rays reveal the clinical course of contained rupture of thoracic mycotic aneurysm. HD5 chest X-ray reveals a large pleural effusion, which was exudate but not blood. HD6, postTEVAR chest X-ray shows a large hemothorax causing mediastinal shift. HD, hospital day; TEVAR, thoracic endovascular aortic repair; VATS, video-assisted thoracic surgery.

\section{References}

1. Hsu RB, Lin FY. Infected aneurysm of the thoracic aorta. J Vasc Surg 2008;47:270-6.

2. Muller BT, Wegener OR, Grabitz K, Pillny M, Thomas L, Sandmann W. Mycotic aneurysms of the thoracic and abdominal aorta and iliac arteries: Experience with anatomic and extra-anatomic repair in 33 cases. J Vasc Surg 2001;33:106-13.

3. Hirano K, Tokui T, Nakamura B, Inoue R, Inagaki M, Toyoshima H, et al. Hybrid therapy for mycotic aortic aneurysm with stent-graft and video-assisted thoracoscopic debridement. Ann Vasc Dis 2019;12:69-73.

4. Sorelius K, Wanhainen A, Wahlgren CM, Langenskiold M, Roos H, Resch T, et al. Nationwide study on treatment of mycotic thoracic aortic aneurysms. Eur J Vasc Endovasc Surg 2019;57:239-46.

5. Stellmes A, Allmen RV, Derungs U, Dick F, Makaloski V, Do DD, et al. Thoracic endovascular aortic repair as emergency therapy despite suspected aortic infection. Interact Cardiovasc Thorac Surg 2013;16:45964.

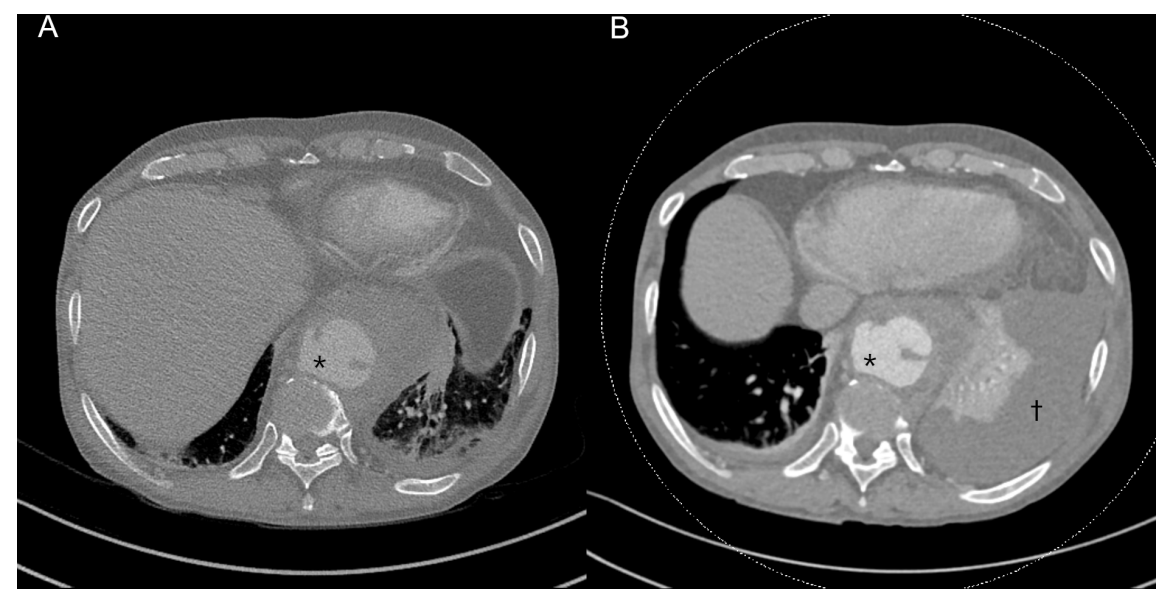




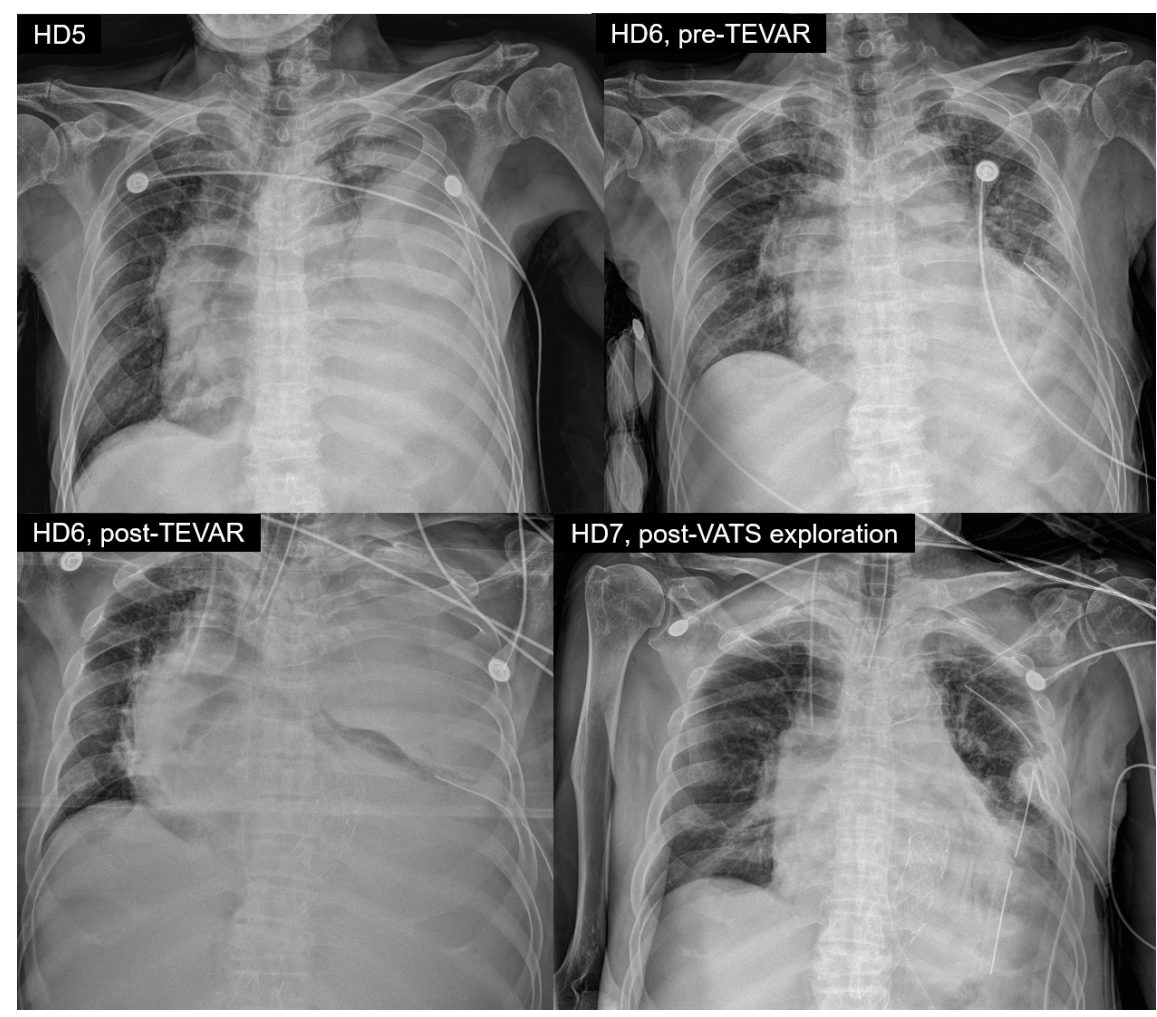

